ORIGINAL ARTICLE

DOI: $10.2478 / \mathrm{ffp}-2020-0002$

\title{
Micropropagation and in vitro rooting of Robinia pseudoacacia L. recalcitrant genotypes
}

\author{
Iwona Szyp-Borowska ${ }^{1} \bowtie$, Joanna Ukalska ${ }^{2}$, Tomasz Wojda ${ }^{1}$, Małgorzata Sułkowska ${ }^{1}$, \\ Marcin Klisz ${ }^{1}$ \\ ${ }^{1}$ Forest Research Institute, Department of Silviculture and Genetics of Forest Trees, Braci Leśnej 3, Sękocin Stary, \\ 05-090 Raszyn, Poland, e-mail: I.Szyp@ibles.waw.pl \\ ${ }^{2}$ Warsaw University of Life Sciences, Institute of Forest Sciences, Department of Forest Management Planning, \\ Dendrometry and Forest Economics, Laboratory of Dendrometry and Forest Productivity, Nowoursynowska 159, \\ 02-776 Warsaw, Poland
}

\begin{abstract}
In forest production, there is an emerging tendency towards the planting of fast-growing trees as attractive, renewable energy sources. Hence, efforts were made to develop a method of micropropagation by organogenesis of seven clones of black locust (Robinia pseudoacacia L.) that are resistant to propagation by traditional vegetative methods, as well as one plus tree (no. 9755) at the age of 60, to see if the age of the mother plant is a limitation in the micropropagation of black locust trees. Overall results suggest that Murashige and Skoog medium supplemented with $30 \mathrm{~g} \mathrm{l}^{-1}$ sucrose, $0.6 \mathrm{mg}^{-1}$ 6-benzylaminopurine (BAP) and $0.1 \mathrm{mg} \mathrm{l}^{-1}$ naphthalene acetic acid (NAA) is better for the propagation of each genotype of $R$. pseudoacacia than Woody Plant Medium with the same growth regulators, and the age of the donor plant does not affect the organogenic potential. Recalcitrance to adventitious rooting from adventitious shoot formation is a major limitation for the clonal micropropagation of forest trees. Our results showed that although the roots were also formed spontaneously in the growth medium without growth hormones for the tested black locust clones, the application of auxin increased the total root length compared to that in the medium with active carbon and control. A significant effect of the additives of hormone and sucrose on the total root length was found. Increasing the sucrose concentration stimulated the induction of roots in each of the tested concentrations $\left(5,10,15\right.$ or $\left.20 \mathrm{~g}^{-1}\right)$. Additionally, the change in sugar dose in the rooting medium caused significant differences in total root length.
\end{abstract}

\section{KEY WORDS}

black locust, in vitro cultures, medium effect

\section{INTRODUCTION}

The black locust (Robinia pseudoacacia L.) has been present in Poland for more than 200 years, and its range now covers the whole country, with the highest concen- tration of occurrence found in the western region. The intensive growth at an early age and the possibility of growth on poor soils, as well as the production of valuable timber, have caused a trend in breeding the species on fast-growing tree plantations. So far, in Poland, as 
a result of breeding activities, 3 selected seed stands, 44 plus trees and 2 seed orchards have been planted. Recent investigations carried out at the Forest Research Institute enabled localisation of a few previously unknown black locust tree stands characterised by an exceptionally straight form of trunk. From these trees, root cuttings were sampled and seedlings were obtained, which can create the basis of the most valuable tree stands of black locust in Poland. Breeding of selected clones of R. pseudoacacia, which are characterised by desired features, seems to be promising for the production of high-quality wood. Unfortunately, the traditional vegetative propagation methods turn out to be ineffective for particular genotypes. In cases where propagation is ineffective or even impossible, it is advantageous to use micropropagation techniques (tissue culture) (Rédei et al. 2002). This is, due to the high rate of multiplication, an attractive method for the propagation of elite genotypes. In this field, considerable knowledge has been accumulated on the basis of investigations performed by Mascarenhas et al. (1982), Chalupa (1983), Barghchi (1987) and Arrillaga (1993). The research on micropropagation of black locust trees was initiated in the 1980s. The first data came from Romania (Enescu and Jucan 1985). In the same year, Balla and Vértesy (1985) reported on the success of micropropagation of black locust trees in Hungary. In spite of several protocols for regeneration being reported, the regeneration efficiency has been shown to be influenced by explant genotypes. The crucial success of this process is the composition and concentration of micro- and macroelements, plant growth regulators (PGRs) and other components in the culture medium. Murashige and Skoog (1962) media (MS) and Woody Plant Medium (WPM) (McCown and Lloyd 1981) are widely used in tissue culture. Both these media have been reported to be used in the in vitro breeding of many trees, including the black locust (Chalupa 1983; Han and Park 1999; Vieitez et al. 1985). However, the application of large-scale micropropagation of $R$. pseudoacacia $\mathrm{L}$. is limited by the rooting of new shoots. A good rooting system is a prerequisite for the survival of in vitrogrown plantlets in the field (Benková and Bielach 2010). The physiological and biochemical qualities of mother plants, such as their age and genetic makeup, could limit rooting performance of cuttings derived from them (Hartmann et al. 1990). Therefore, research on adventitious root formation is highly important from a practi- cal point of view. Although auxins are the main factors involved in the induction of rooting, other endogenous and exogenous substances also influence the rhizogenesis process. Moncousin (1991) has discussed factors such as the basal medium concentration, carbohydrate nutrition, light, darkness, temperature and the presence of activated charcoal (AC).

The aim of this research was to develop an in vitro propagation method for black locust that is suitable for large-scale cloning of genotypes that are recalcitrant to vegetative propagation by traditional methods.

\section{Material AND Methods}

\section{Plant material and culture condition}

The experiments were undertaken with five selected 10-year-old clones of R. pseudoacacia (Tab. 1) and one plus tree at the age of 60 . In vitro culture was initiated using dormant axillary buds sampled in the month of February from the trees growing in the seed orchards and forest stands in Krosno Forest District in western Poland. The shoots were grown in a phytotron at $25^{\circ} \mathrm{C}$ day temperature and $20^{\circ} \mathrm{C}$ night temperature with a 16-h photoperiod until the buds developed. The light intensity was approximately $30-40 \mu \mathrm{mol} \mathrm{m}^{-2} \mathrm{sec}^{-1}$, and the humidity was maintained at $70 \%$.

Table 1. List of propagated genotypes of Robinia pseudoacacia L. and a summary of the number of received rooted cuttings

\begin{tabular}{|l|c|}
\hline Genotype number & No. of seedlings from in vitro cultures \\
\hline 9755 (grafted) & 265 \\
\hline 9759 (grafted) & 68 \\
\hline 9757 (root cuttings) & 247 \\
\hline 9735 (grafted) & 80 \\
\hline 9758 (grafted) & 212 \\
\hline 9755 plus tree & 139 \\
\hline
\end{tabular}

\section{Culture establishment}

The shoots developed from buds were used as the starting material for micropropagation. The shoots (primary explants) were sterilised in $0.1-0.2 \%$ mercuric chloride solution and transferred to MS medium (Murashige and Skoog 1962) or WPM (McCown and Lloyd 1981) 
Table 2. The composition of culture medium for vegetative propagation of Robinia pseudoacacia L.

\begin{tabular}{|l|c|c|c|}
\hline Compounds in & Initiation & Proliferation & $\begin{array}{c}\text { Induction } \\
\text { of roots }\end{array}$ \\
\hline MS medium & $\begin{array}{c}\text { full } \\
\text { strength }^{\mathrm{a}}\end{array}$ & $\begin{array}{c}\text { half } \\
\text { strength }^{\mathrm{a}}\end{array}$ & $\begin{array}{c}\text { half } \\
\text { strength }^{\mathrm{a}}\end{array}$ \\
\hline Sucrose $\left(\mathrm{g} \mathrm{l}^{-1}\right)$ & 30 & 30 & $5,10,15,20$ \\
\hline Agar $\left(\mathrm{g} \mathrm{l}^{-1}\right)$ & 5.5 & 5.5 & 5.5 \\
\hline BAP $\left(\mathrm{mg} \mathrm{l}^{-1}\right)$ & 0.6 & 1 & - \\
\hline NAA $\left(\mathrm{mg} \mathrm{l}^{-1}\right)$ & 0.1 & - & 0.2 \\
\hline IBA $\left(\mathrm{mg} \mathrm{l}^{-1}\right)$ & - & - & $(2.0)^{\mathrm{b}}$ \\
\hline $\begin{array}{l}\text { Activated } \\
\text { charcoal }\left(\mathrm{g} \mathrm{l}^{-1}\right)\end{array}$ & - & 5.8 & 5.8 \\
\hline pH & 5.8 & & \\
\hline
\end{tabular}

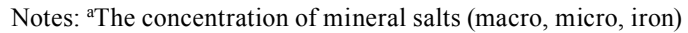

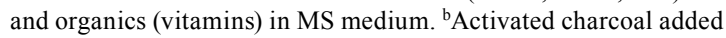
optionally.

Key: BAP, 6-benzylaminopurine; IBA, indolyl-3-butyric acid; MS, Murashige and Skoog; NAA, naphthalene acetic acid.

supplemented with $30 \mathrm{~g} \mathrm{l}^{-1}$ sucrose, $0.6 \mathrm{mg} \mathrm{l}^{-1}$ 6-benzylaminopurine (BAP), $0.1 \mathrm{mg}^{-1}$ naphthalene acetic acid (NAA) and $5.5 \mathrm{mg} \mathrm{l}^{-1}$ agar. The first experiment was conducted to study the effect of basal salt medium on the process of multiplication of black locust clones. After 4 weeks, the fresh weight (FW) of the individual shoots was calculated. New adventitious shoots of approximately $1.5-3 \mathrm{~cm}$ in length were subcultured in proliferation MS medium that was diluted to half and supplemented with $1 \mathrm{mg} \mathrm{l}^{-1}$ BAP (Tab. 2). The proliferated shoots were elongated on a growth medium without growth hormone $(1 / 2 \mathrm{MS} 0)$. This procedure was repeated many times to obtain the desired number of cuttings.

\section{In vitro rooting and acclimatisation}

In vitro the shoots were transferred to $1 / 2 \mathrm{MS}$ without growth hormone supplementation but with activated carbon at $2 \mathrm{~g} \mathrm{l}^{-1}$ for 1 week; then, for 4-5 weeks, they were transferred to rooting medium supplemented with indolyl-3-butyric acid (IBA) at $0.2 \mathrm{mg} \mathrm{l}^{-1}$. To optimise the rooting conditions, in the second experiment, the effects of different concentrations of sucrose $(5,10,15$, $20 \mathrm{~g}^{-1}$ ) and $\mathrm{AC}$ supplementation were tested. In this experiment, explants were cultured on media containing mineral salts according to half strength MS media. Each treatment included six replications, and each replicate included five explants. Explants cultured on half strength MS medium without hormones but with sucrose at $10 \mathrm{~g} \mathrm{l}^{-1}$ were used as a control. The following traits were evaluated after 7 and 14 days: number of adventitious roots per explant (RN), total root length (TRL) and rooting percentage (R). Taking into account the number of received adventitious shoots, each experiment was carried out with a different number of black locust genotypes. In the first experiment, all genotypes were compared. In Model 2, clones 9755 (PT), 9757, 9758 and 9755 were taken into consideration, and in Model 3, clone 9758 was used.

After 4 weeks, plants with well-developed roots were transferred for acclimatisation into plastic pots on substrate peat and perlite in the ratio 1:2 and covered with foil. After 7-10 days, air humidity was gradually reduced. The cover was regularly opened once per day for $2 \mathrm{~h}$. After the cover was removed, the plants were left in a greenhouse until lignification. Regeneration rate was scored by the number of plants for each clone that developed stems and roots.

\section{Statistical analyses}

Effect of basal medium

To investigate the effect of the medium and genotype on the FW gain, a linear model was used (Model 1):

$$
\mathrm{FW}_{i j k}=m+\mathrm{M}_{i}+\mathrm{G}_{j}+\mathrm{MG}_{i j}+e_{i j k}
$$

where:

$\mathrm{FW}_{i j k}$ - the FW gain for the $i$-th medium $(i=1,2)$ and $j$-th genotype $(j=1, \ldots, 6)$, for $k$-th explant,

$m$ - the overall mean,

$\mathrm{M}_{i}$ - the main effect of the $i$-th medium,

$\mathrm{G}_{j} \quad$ - the main effect of the $j$-th genotype,

$\mathrm{MG}_{i j}$ - the medium $\times$ genotype interaction effect,

$e_{i j k} \quad-$ an experimental error effect.

The FW data were not normally distributed (Shapiro-Wilk test); therefore, the analysis of variance according to Model 1 was made on log-transformed data.

\section{Effect of sucrose concentration}

To determine the effect of the genotype and sucrose concentration in the medium on the rooting of seedlings after 7 and 14 days, we used a repeated measures analysis of variance according to the following linear model (Model 2):

$$
\mathrm{R}_{i j k l}=m+\mathrm{SC}_{i}+\mathrm{G}_{j}+\mathrm{T}_{k}+\mathrm{SCT}_{i k}+\mathrm{GT}_{j k}+e_{i j k l,}
$$


where:

$\mathrm{R}_{i j k l} \quad$ - rooting for the $i$-th concentration of sucrose $(i=1, \ldots, 5)$ and $j$-th genotype $(j=1, \ldots, 4)$ in $k$-th time of rooting process $(k=1,2)$ in $l$-th replicate $(l=1, \ldots, 6)$,

$\mathrm{SC}_{i} \quad$ - the main effect of the $i$-th concentration of sucrose,

$\mathrm{T}_{k} \quad$ - the main effect of $k$-th time of rooting process, $\mathrm{SCT}_{i k}$ - the concentration of sucrose $\times$ time of rooting process interaction effect,

$\mathrm{GT}_{j k} \quad$ - the genotype $\times$ time of rooting process interaction effect,

and the meaning of other parameters is analogous to that of Model 1.

Effect of supplements in rooting medium

The TRL was strongly positively correlated with the number of roots RN. Therefore, the RN variable was included as a covariance variable in the models for analysis of the TRL variable.

To analyse the effect of active carbon (AC) supplementation and sucrose dose on TRL in the basic rooting medium, the following general linear model (Model 3) was applied:

$$
\mathrm{TRL}_{i j k}=m+\mathrm{A}_{i}+\mathrm{SD}_{j}+\mathrm{RN}_{i j k}+e_{i j k}
$$

where:

$\mathrm{TRL}_{i j k}$ - the sum of root length for the $i$-th additive in medium $(i=1,2,3)$ and $j$-th sucrose dose ( $j=1,2,3)$ for the $k$-th seedling,

$\mathrm{A}_{i} \quad-$ the main effect of the $i$-th additive (AC, growth hormone and control) in the medium,

$\mathrm{SD}_{j} \quad-$ the main effect of the $j$-th sucrose dose (constant, increase, decrease),

$\mathrm{RN}_{i j k}$ - the number of roots as a covariate, and the meaning of other parameters is analogous to Model 1.

The TRL response distribution was not normal; therefore, analyses of variance were made on cube roottransformed data.

For significant effects in models $1-3$, pairwise comparisons were made between the least square means with Tukey's post hoc test and the Tukey-Kramer correction for unequal sample sizes. All calculations according to models 1-3 were performed using the GLM procedure of SAS/STAT ${ }^{\circledR}$ v. 14.3 (SAS Institute Inc., 2017).

\section{Results}

\section{Culture induction and proliferation}

The breeding cycle, from the initiation of cultures to availability of black locust plants capable of growth in natural conditions, took an average of 5-6 months (April to September). The efficiency of the method is characterised by the number of obtained seedlings rooted cuttings for each clone (Tab. 1). Unfortunately, for the 9759 clone, only several dozen seedlings rooted cuttings were obtained due to the difficulties in rooting.

\section{Effect of basal medium}

A significant effect of the medium type on the fresh mass gain of Robinia clones was found (Tab. 3, Model 1). Furthermore, the medium $\times$ genotype interaction was significant. The genotypes 9759 and 9758 showed significantly better results on MS medium than on WPM medium; however, in the other genotypes, the preference of the particular medium was not significant (Fig. 1). Microshoots of 9757 grown on MS or WPM had a higher FW than those of 9735.

Table 3. Results of analyses of variance according to models 1-3

\begin{tabular}{|l|r|r|r|r|c|}
\hline \multicolumn{1}{|c|}{ Effect } & DF & \multicolumn{1}{c|}{ SS } & \multicolumn{1}{c|}{ MS } & F & P \\
\hline \multicolumn{7}{|c|}{ Model 1 } \\
\hline Genotype G & 5 & 23.44 & 4.69 & 20.99 & $<0.001$ \\
\hline Medium M & 1 & 6.31 & 6.31 & 28.24 & $<0.001$ \\
\hline $\mathrm{M} \times$ G & 5 & 2.89 & 0.58 & 2.59 & 0.026 \\
\hline \multicolumn{7}{|c|}{ Model 2} \\
\hline SC & 4 & $2,710.65$ & 677.66 & 8.08 & 0.002 \\
\hline Genotype G & 3 & $4,959.32$ & $1,653.11$ & 19.71 & $<0.001$ \\
\hline Time T & 1 & $3,461.65$ & $3,461.65$ & 68.03 & $<0.001$ \\
\hline T $\times$ SC & 4 & 390.16 & 97.54 & 1.92 & 0.172 \\
\hline T $\times$ G & 3 & 215.76 & 71.92 & 1.41 & 0.287 \\
\hline \multicolumn{7}{|c|}{ Model 3 } \\
\hline Additives A & 2 & $43,954.03$ & $21,977.02$ & 31.12 & $<0.001$ \\
\hline SD & 2 & $18,932.45$ & $9,466.23$ & 13.41 & $<0.001$ \\
\hline RN & 1 & $247,560.65$ & $24,7560.65$ & 350.60 & $<0.001$ \\
\hline
\end{tabular}

Key: DF, degrees of freedom; F, test statistic; MS, mean square; P, probability; RN, root number; $\mathrm{SC}$, sucrose concentration; SD, sucrose dose; SS, sum of squares.

FW on MS medium for clone 9755 (PT plus tree) was significantly higher than for clone 9735 and signifi- 
cantly lower than for clone 9757, but was not different from the FW obtained for other clones. In conclusion, the age of the donor plant did not affect the organogenic potential of $R$. pseudoacacia.

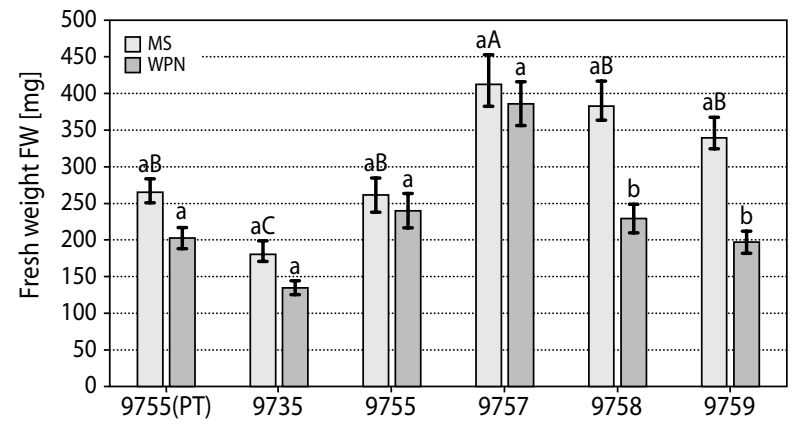

Figure 1. Means of FW gain for the studied genotypes of Robinia pseudoacacia with two variants of medium (bars are standard errors; lower case letters indicate a comparison of the media for a clone; upper case letters indicate a comparison of the 9755 [PT plus tree] clone with other clones on MS medium; means with the same letter are not significantly different at $p \leq 0.05$ )

Key: FW, fresh weight; MS, Murashige and Skoog; WPM, Woody Plant Medium.

The first proliferation was observed within 12-14 days after placing black locust explants on the medium. At the bases of shoots, adventitious buds were observed, which were grown from a mass of callus cells (indirect organogenesis with callus stage). New adventitious shoots, where the number for one explant ranged from 8 to 30 pieces and even more, were formed (Fig. 2A).

\section{In vitro rooting and acclimatisation}

Effect of sucrose concentration

The process of rooting of adventitious shoots constituted 2-3 weeks, although on some shoots, the formation of roots was observed after 7 and 14 days only when they were transferred to medium. Based on the analysis of variance, significant differences were detected among genotypes, sucrose concentration and time (Tab. 3, Model 2). The lowest percentage of rooted plants (6.7\%) was found for the 9759 clone, while the 9755 clone was characterised by a significantly higher percentage of rooting $(42.4 \%)$.

Increasing the sucrose concentration stimulated the induction of roots (Fig. 3). The rooting percentage was higher when a higher concentration of sucrose was used

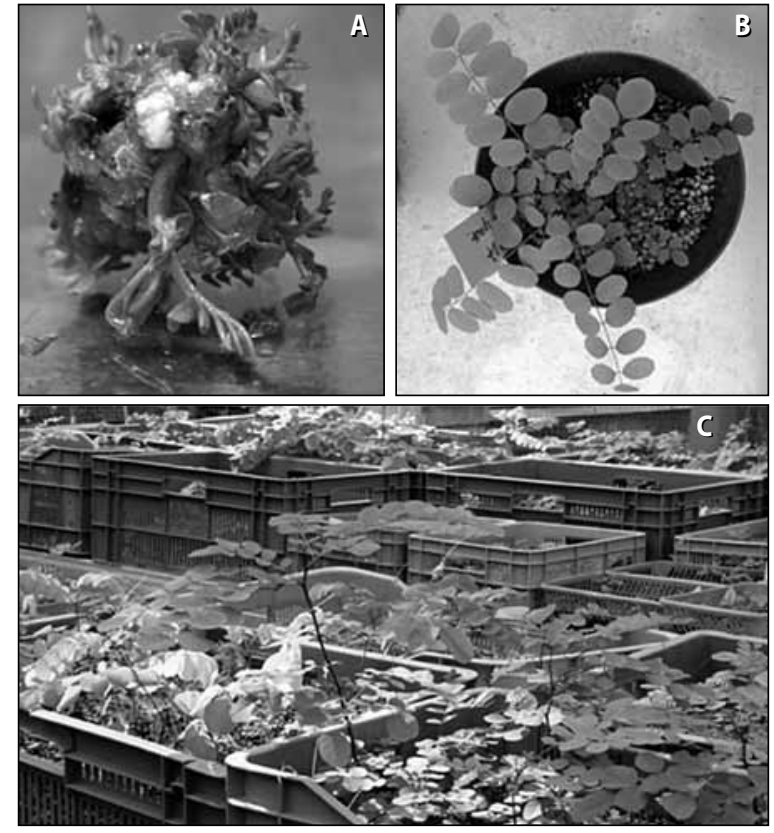

Figure 2. (A) Formation of buds and adventitious shoots at the bases of black locust explants in vitro. (B) An in vitroobtained and ex vitro-adapted black locust plant at the age of 1 year. (C) In vitro-obtained and ex vitro-adapted black locust plants at the age of 2 years

in the induction phase. The addition of sucrose in each of the tested concentrations resulted in a significant increase in the percentage of rooted plants in comparison to the sucrose-free variant.

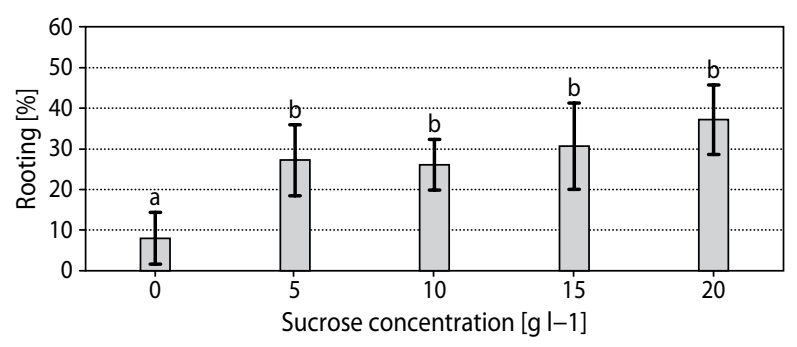

Figure 3. Mean percentage of rooted plantlets for the sucrose concentration variants in the medium (bars are standard errors; means with the same lower case letter are not significantly different at $p \leq 0.05$ )

Effect of supplements in the rooting medium

A significant effect of the additives on TRL was found (Tab. 3, Model 3). The application of auxin increased TRL compared to the medium with AC and the control 
(C) (Fig. 4). However, the number of induced roots did not change; only their length was increased. Additionally, the change in sugar concentration in the rooting medium caused significant differences in TRL. The TRL was lower for the clones that were transferred to the rooting medium at lower sucrose concentrations than for those on the medium from the previous passage. However, the transfer of clones to the medium with a higher concentration of sucrose did not cause TRL growth.

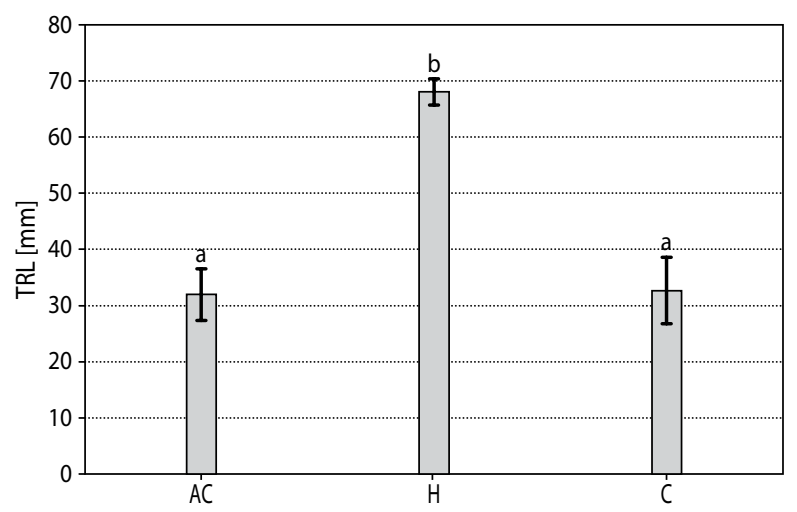

Figure 4. TRL means, corrected due to the RN covariance variable, for media additives (bars are standard errors; means with the same lower case letter are not significantly different at $p \leq 0.05$ )

Key: AC, active carbon; C, control; H, hormone; RN, root number; TRL, total root length.

At the end of August, rooted cuttings, obtained through in vitro culture and adapted ex vitro, were transferred outside the greenhouse to enable them to lignify before the winter (Fig. 2B). Two-year-old plants of black locust were used as supplemental material in the seed orchard (Fig. 2C).

\section{Discussion}

Micropropagation (vegetative reproduction in vitro) allows relatively fast and effective reproduction of individuals selected in terms of growth characteristics or quality of wood. It also provides the possibility to obtain the progeny of trees resistant to traditional methods of vegetative propagation (e.g., rooting of root cuttings, shoots or grafting). According to Redei et al. (2002), the efficiency of the micropropagation method allows for the production of 10,000 microplants in 1 year from 100 shoots (primary explants), of which $80 \%$ will be acclimated successfully. Good results from this process depend on the medium composition, growth regulators and other components in the culture medium. Our results showed that the most suitable medium for the in vitro cultivation of black locust clones was MS. Similarly, good results for MS medium were reported by Han and Keathley (1989), Chalupa (1983) and Zhang et al. (2007). The MS medium is widely used in tissue cultures and is considered to be rich in substrates. In comparison to MS medium, WPM is characterised by a reduced overall content of salts, in particular, nitrogen and chlorine compounds.

Another important factor determining the success of the method is the selection of growth regulators at particular stages of cultivation and the appropriate concentration of sucrose. Generally, the cytokinin BAP is used, which, in combination with NAA, can promote induction of adventitious buds. In the case of propagation of black locust clones, it was observed that BAP at a concentration of $0.6 \mathrm{mg} 1^{-1}$ and NAA at a concentration of $0.1 \mathrm{mg} 1^{-1}$ in the medium led to induction of organogenesis, while the presence of BAP at a concentration of $1 \mathrm{mg} 1^{-1}$ promoted the proliferation of adventitious buds. Chalupa (1983) used BAP at a concentration at $0.4-0.6 \mathrm{mg}^{-1}$ and IBA at a concentration of $0.05 \mathrm{mg}^{-1}$ and obtained a high rate of black locust shoot multiplication. Davis and Keathley $(1987,1992)$ and Han et al. (1990) used MS medium supplemented only with BAP, which promoted the prolongation of the adventitious shoots of black locust, while a higher level of BAP led to the formation of numerous adventitious shoots and callus. There are several factors that restrict the vegetative propagation responses, including the age of the donor plant. In vitro clonal propagation of a 300-year-old black locust tree was achieved by Gyulai (2010) using aseptic bud cultures and was followed by shoot micropropagation, and the age of the donor plant did not affect the organogenic potential of explants. Our results with a 60 -year-old plus tree of Robinia confirm these observations.

The next step in tissue culture is rhizogenesis, which, similar to the induction and propagation of adventitious buds, is a complex morphogenetic process dependent on the activity of growth regulators. The promoters of root initiation are auxins, which stimulate 
cells to form meristems (García-Garrido et al. 2000). Rooting recalcitrance in a difficult-to-root species of Eucalyptus was found to be associated with changes in auxin concentration and sensitivity during rooting (Aumond et al. 2017). The most commonly used auxins are indolyl-3-acetic acid (IAA), IBA and NAA. For black locust tree, the investigations of Kovačević et al. (2014) showed that NAA stimulated root formation better than IBA. Most of the supplied auxins act at the wound site, inducing cell dedifferentiation and leading to a new root meristem later on. However, in our experiment, auxin treatment did not increase the number of roots, but only their mean length (TRL $-68 \mathrm{~mm}$ ). We observed that, for the tested black locust clones, also formed the roots spontaneously in the growth medium with a higher sucrose concentration or active charcoal. The phenomenon of this activity of sugar and activated carbon associated with the formation of adventitious roots was observed in many studies (Bousselmame et al. 2001; Damiano 1978; Dumas and Monteuuis 1995; Fridborg and Eriksson 1975). Carbohydrates contribute to the formation of adventitious roots by supplying energy and carbon necessary for cell division, establishing new root meristems, and by root formation itself (Druege 2009). Similarly, a higher content of soluble sugars and starch in the rooting medium was associated with higher rooting response in Tectona grandis cuttings (Husen and Pal 2007). The effect of activated carbon activity is connected to the formation of a darkened environment, adsorption of undesirable and inhibiting substances and binding of growth regulators and other organic compounds, which promotes changes that lead to the formation of roots (Ahuja 1985; Bousselmame et al. 2001; Dumas and Monteuuis 1995; Pan and Staden 1998). Root organogenesis process is directly related to the stage of adaptation of in vitro culture seedlings to growth and development in natural conditions. It requires multiple treatments, and it gives an essential meaning to the application possibility of the method of in vitro propagation on a large scale. In natural conditions of growth, the assimilation apparatus of seedlings must undergo reconstruction and adaptation. The maintenance of low light intensity and high humidity in the air during the first weeks of growth of seedlings after transplanting reduce their loss of water. The black locust seedlings were grown in a greenhouse for a period of 3 months, where, over time, the humidity was lowered, while the intensity of light was increased. The elaborated method of in vitro multiplication of black locust was proven to be effective, although, in the case of two clones, 9733 and 9735 , the rooting process required additional treatments. The transfer of shoots to the substrate without auxins, but with supplementation of the medium with AC turned out to be favourable. In particularly valuable genotypes, the vegetative progeny can be utilised as a source of seeds with an elevated genetic value or as a material for plantations of special purpose, for example, fast-growing plantations.

\section{Conclusions}

The developed method for vegetative propagation of $R$. pseudoacacia L. allows obtaining reproductive material. The most suitable medium for the studied clones was the one developed by Murashige and Skoog (1962). BAP at a concentration of $0.6 \mathrm{mg} 1^{-1}$ and NAA at a concentration of $0.1 \mathrm{mg} 1^{-1}$ in the medium led to the induction of organogenesis, whereas the presence of BAP at a concentration of $1 \mathrm{mg} 1^{-1}$ led to the proliferation of adventitious buds. Although the roots formed spontaneously in the growth medium without growth hormones, supplementation with hormones stimulated the formation of roots on the adventitious shoots of the tested black locust clones. In many rooting recalcitrant species, application of exogenous auxin or increasing the sucrose concentration are needed to achieve rooting responses .

\section{References}

Ahuja, M.R. 1985. Somaclonal variation. In: Cell and tissue culture in forestry. Vol. 1. General principles and biotechnology (eds.: J.M. Bonga, O.J. Durzan). Martinus Nijhoff Publisher, Dordrecht, The Netherlands, 272-285.

Arrillaga, I. 1993. Regenerating plants from in vitro culture of black locust cotyledon and leaf explants. Hortscience, 28, 942-945. DOI: doi.org/10.21273/ HORTSCI.28.9.942

Aumond, M.L. et al. 2017. Events associated with early age-related decline in adventitious rooting competence of Eucalyptus globulus Labill. Frontiers 
in Plant Science, 8, 1734. DOI: doi.org/10.3389/ fpls.2017.01734

Balla, I., Vértesy, J. 1985. Experiences and problems related to the micropropagation of black locust. In: Symposium on In Vitro Problems Related to Mass Propagation of Horticultural Plants, Book of Abstracts II. International Society of Horticultural Science, Gembloux, Belgium.

Barghchi, M. 1987. In vitro mass clonal propagation of Robinia pseudoacacia (Black locust) cv "Jaszkiseri”. Plant Science, 53, 183-189.

Benkova, E., Bielach, A. 2010. Lateral root organogenesis - from cell to organ. Current Opinion in Plant Biology, 13, 677-683. DOI: 10.1016/j.pbi.2010.09.006

Bousselmame, F., Kenny, L., Chlyah, H. 2001. Optimization of culture conditions for in vitro rooting of argan (Argania spinosa L.). Comptes Rendus de l'Académie des Sciences - Series III - Sciences de la Vie, 324 (11), 995-1000.

Chalupa, V. 1983. Micropropagation of conifer and broadleaved forest trees. Commun. Inst. Forest Cech., 13, 7-39.

Damiano, C. 1978. Il carbone attivo nella coltura in vitro della fragola. Frutticoltura, 40, 49-50.

Davis, J.M., Keathley, D.E. 1987. Differential responses to in vitro bud culture in mature Robinia pseudoacacia L. (black locust). Plant Cell Reports, 6, 431-434.

Davis, J.M., Keathley, D.E. 1992. Micropropagation of black locust (Robinia pseudoacacia). Biotechnology in Agriculture and Forestry, 18, 25-39.

Druege, U. 2009. Involvement of carbohydrates in survival and adventitious root formation of cuttings within the scope of global horticulture. In: Adventitious root formation of forest trees and horticultural plants - from genes to applications (eds.: K. Niemiand, C. Scagel). Research Signpost, Kerala, 187-208.

Dumas, E., Monteuuis, O. 1995. In vitro rooting of micropropagated shoots from juvenile and mature Pinus pinaster explants - influence of activated charcoal. Plant Cell, Tissue and Organ Culture, 40, 231-235.

Enescu, V., Jucan, A. 1985. Problems of the in vitro micropropagation of black locust (Robinia pseudoacacia L.). In: New Ways in Forest Genetics. Proceeding of the 20th Canadian Tree Improvement Asso- ciation Meeting, Quebec (eds.: F. Caron, A.G. Corriveau, T.J.B. Boyle), 179-184.

Fridborg, G., Eriksson, T. 1975. Effects of activated charcoal on morphogenesis in plant tissue cultures. Physiologia Plantarum, 34, 306-308. DOI: doi. org/10.1111/j.1399-3054.1975.tb03843.x

García-Garrido, J.M., Tribak, M., Rejon-Palomares, A., Ocampo, J.A., García-Romera, I. 2000. Hydrolytic enzymes and ability of arbuscular mycorrhizal fungi to colonize roots. Journal of Experimental Botany, 51, 1443-1448. DOI: doi.org/10.1093/ $\mathrm{jxb} / 51.349 .1443$

Han, H.K., Keathley, D.E. 1989. Regeneration of whole plants from seedling-derived callus of black locust (Robinia pseudoacacia L.). Nitrogen Fixing Tree Research Reports, 7, 129-131.

Han, H.K., Davis, J.N., Keathley, D.E. 1990. Different responses persist in shoot explants regenerated from callus of two mature black locust tree. Tree Physiology, 6, 235-240.

Han, K.H., Park, Y.G. 1999. Somatic embryogenesis in black locust (Robinia pseudoacacia L.). Somatic Embryogenesis in Woody Plants, 5, 149-161.

Hartmann, H.T., Kester, D.E., Davies, F.T. Jr. 1990. Plant propagation: principles and practices. Prentice Hall, Englewood Cliffs, New Jersey.

Husen, A., Pal, M. 2007. Metabolic root primordium development in changes during adventitious Tectona grandis Linn. f. (teak) cuttings as affected by age of donor plants and auxin (IBA and NAA) treatment. New Forests, 33, 309-323. DOI: doi.org/10.1007/ s11056-006-9030-7

Kovačević, B.Z. et al. 2014. Naphtalenic acids - alternative rooting stimulators in black locust microroots. Matica Srpska Journal for Natural Sciences, 126, 7-13.

Mascarenhas, A.F., Hazra, S., Potelar, U., Kulkarni, D.K., Gupta, P.K. 1982. Leguminous trees. In: Plant tisuue culture. Proceedings $5^{\text {th }}$ International Congress of Plant Tissue and Cell Culture, Tokyo (ed.: A. Fujiwara), 719.

McCown, B.H., Lloyd, G. 1981. Woody Plant Medium (WPM) - a mineral nutrient formulation for microculture of woody plant species. HortScience, 16, 453-453.

Moncousin, Ch. 1991. Rooting of microcuttings: fundamental aspects. Acta Horticulturae, 289, 311-317. 
Murashige, T., Skoog, F. 1962. A revised medium for rapid growth and bioassays with tobacco tissue cultures. Physiologia Plantarum, 15, 473-497.

Pan, M.J., Staden, J. 1998. The use of charcoal in in vitro culture - A review. Plant Growth Regulation, 26, 155-163.

Rédei, K., Osváth-Bujtás, Z., Balla, I. 2002. Clonal approaches to growing black locust (Robinia pseudoacacia) in Hungary: a review. Forestry, 75 (5), 547-552. DOI: doi.org/10.1093/forestry/75.5.547
SAS Institute. 2017. Inc. SAS/STAT 14.3 User's Guide; SAS Institute Inc.: Cary, NC, USA.

Vieitez, A.M., San-Jose, M.C., Vieitez, E. 1985. In vitro plantlet regeneration from juvenile and mature Quercus robur L. Journal of Horticultural Science, 60 (1), 99-106.

Zhang, J., Liu, Y., Wang, H. 2007. Micropropagation of black locust (Robinia pseudoacacia L.). In: Protocols for Micropropagation of Woody Trees and Fruits (eds.: S.M. Jain, H. Häggman). Springer, 193-199. 